\title{
The full spectral radiative properties of Proxima Centauri ${ }^{\star}$
}

\author{
Ignasi Ribas ${ }^{1}$, Michael D. Gregg ${ }^{2}$, Tabetha S. Boyajian ${ }^{3}$, and Emeline Bolmont ${ }^{4}$ \\ ${ }^{1}$ Institut de Ciències de l'Espai (IEEC-CSIC), C/Can Magrans, s/n, Campus UAB, 08193 Bellaterra, Spain \\ e-mail: iribas@ice.cat \\ 2 Department of Physics, University of California, Davis, One Shields Avenue, Davis, CA 95616, USA \\ 3 Department of Physics \& Astronomy, Louisiana State University, Baton Rouge, LA 70803, USA \\ ${ }^{4}$ Laboratoire AIM Paris-Saclay, CEA/Irfu Université Paris-Diderot CNRS/INSU, 91191 Gif-sur-Yvette, France
}

Received 9 February 2017 / Accepted 26 April 2017

\begin{abstract}
Context. The discovery of Proxima b, a terrestrial temperate planet, presents the opportunity of studying a potentially habitable world in optimal conditions. A key aspect in the modeling of its habitability is to understand the radiation environment of the planet in the full spectral domain.

Aims. We aim to characterize the X-rays to mid-IR radiative properties of Proxima with the goal of providing the top-of-atmosphere fluxes on the planet. We also aim at constraining the fundamental properties of the star, namely its mass, radius, effective temperature and luminosity.

Methods. We have employed observations from a large number of facilities and made use of different methodologies to piece together the full spectral energy distribution of Proxima. In the high-energy domain, we payed particular attention to the contributions of rotational modulation, activity cycle, and flares so that the data provided are representative of the overall radiation dose received by the atmosphere of the planet.

Results. We present the full spectrum of Proxima covering 0.7 to $30000 \mathrm{~nm}$. The integration of the data shows that the top-ofatmosphere average XUV irradiance on Proxima b is $0.293 \mathrm{~W} \mathrm{~m}^{-2}$, that is, nearly 60 times higher than Earth, and that the total irradiance is $877 \pm 44 \mathrm{~W} \mathrm{~m}^{-2}$, or $64 \pm 3 \%$ of the solar constant but with a significantly redder spectrum. We also provide laws for the XUV evolution of Proxima corresponding to two scenarios, one with a constant XUV-to-bolometric luminosity value throughout its history and another one in which Proxima left the saturation phase at an age of about 1.6 Gyr and is now in a power-law regime. Regarding the fundamental properties of Proxima, we find $M=0.120 \pm 0.003 M_{\odot}, R=0.146 \pm 0.007 R_{\odot}, T_{\text {eff }}=2980 \pm 80 \mathrm{~K}$, and $L=0.00151 \pm 0.00008 L_{\odot}$. In addition, our analysis reveals a $\sim 20 \%$ excess in the 3-30 $\mu \mathrm{m}$ flux of the star that is best interpreted as arising from warm dust in the system.

Conclusions. The data provided here should be useful to further investigate the current atmospheric properties of Proxima $b$ as well as its past history, with the overall aim of firmly establishing the habitability of the planet.
\end{abstract}

Key words. stars: individual: Proxima Cen - planets and satellites: individual: Proxima Cen b - planets and satellites: atmospheres planets and satellites: terrestrial planets - X-rays: stars - planet-star interactions

\section{Introduction}

The discovery of a terrestrial planet candidate around the nearest star to the Sun, Proxima Centauri (hereafter Proxima), was reported by Anglada-Escudé et al. (2016) and has opened the door to investigating the properties of a potentially habitable planet from nearest possible vantage point. The detailed studies of Ribas et al. (2016) and Turbet et al. (2016) show that Proxima $\mathrm{b}$ is likely to have undergone substantial loss of volatiles, including water, in particular during the first $~ 100-200 \mathrm{Myr}$, when it could have been in a runaway phase prior to entering the habitable zone. Volatile loss processes once inside the habitable zone could have also been at work. The calculations are highly uncertain (cf. Barnes et al. 2016) and reasonable doubt exists as to whether the modeling schemes currently used are adequate. There are numerous examples in the solar system that would contradict the hypothesis of substantial volatile losses in the early stages of its evolution in spite of the Sun being a strong source of high-energy radiation (Marty 2012). The studies

\footnotetext{
* Table 8 is only available at the CDS via anonymous ftp to cdsarc.u-strasbg.fr (130.79.128.5) or via http://cdsarc.u-strasbg.fr/viz-bin/qcat?J/A+A/603/A58
}

carried out thus far conclude that Proxima $b$ is a viable habitable planet candidate because the presence of surface liquid water cannot be ruled out, as the initial amount of water is uncertain and the efficiency of volatile loss processes is poorly known.

A key ingredient for understanding the evolution and current state of the atmosphere of Proxima $b$ is a proper description of the high-energy irradiation. Today, the flux that Proxima $b$ receives in the XUV domain (X-rays to extreme-UV, EUV) is stronger than that received by the Earth by over an order of magnitude and the level of irradiation was probably even stronger in the past. The situation is likely to be quite different in the UV range as Proxima has a significantly lower photospheric temperature than the Sun and therefore a redder emission distribution. UV irradiation has an impact on photolysis processes, as photoabsorption cross sections of abundant molecules peak in the 100-300 nm range (Hudson 1971), and is also of biological interest (Ranjan \& Sasselov 2016). Therefore, the high-energy budget from the X-rays to the UV is important for many aspects related to the study of Proxima $b$, including understanding its atmospheric physical properties, its photochemistry, and even to the first attempts to constrain a putative biosphere on its surface. The optical and IR irradiation, on the other hand, is the main 
contributor to the overall energy budget, thus determining the surface temperature of the planet and, ultimately, its habitability.

Ribas et al. (2016) obtained a rough XUV spectrum of Proxima and also discussed possible XUV evolution laws. Here we generalize this study by providing better estimates of the radiation environment of Proxima $b$ and extending the analysis to the full spectral domain (X-rays to mid-IR). In Sect. 2 we combine observations over a wide wavelength range to deduce the spectral energy distribution (SED) of Proxima that is representative of the average radiation dose. As a consequence of this analysis, we identify a conspicuous IR excess, possibly due to dust in the Proxima system, which is discussed in Sect. 3. Good determinations of the basic physical properties of its stellar host, namely its mass, radius, effective temperature, and bolometric luminosity are also important to understand the climate of Proxima b. In Sect. 4 we use all available observational constraints to provide the best estimate of such properties. In Sect. 5 we address the issue of the XUV evolution law and propose two relationships that take into account the pre-main sequence evolution of Proxima. We have also performed a new calculation of water loss during the early stages of the evolution of Proxima $b$ and we compare the results with our earlier estimates in Ribas et al. (2016). Finally, the conclusions of our work are given in Sect. 6.

\section{Spectral energy distribution}

The aim of the study is to provide the full energy distribution at the top of the atmosphere of Proxima $b$ by characterizing the electromagnetic spectrum of the host star as accurately as possible. This necessarily implies making use of a number of different facilities and also employing theoretical estimates for those wavelength intervals that do not have observations. Some of the datasets that we consider were already discussed in Ribas et al. (2016) and we just give a short description and additional relevant details. We have also improved the methodology in the case of the far-ultraviolet (FUV) range (Sect. 2.5) and this leads to a total integrated XUV flux value that differs by a few per cent from that presented by Ribas et al. (2016). A summary of the wavelength intervals considered and the datasets used is provided in Table 1, and the full details are discussed in the sections below.

One of the complications associated with the determination of the flux emitted by Proxima is the effect of stellar flares. Flare events can significantly increase the flux with a relative contribution that is stronger at shorter wavelengths. In the present study we have estimated the mean XUV flux over a relatively extended timescale in an attempt to measure the overall dose on the planetary atmosphere, including the flare contribution. Our strategy is, thus, to consider long integration times to ensure proper averaging of the flare events with the quiescent flux. We have applied a further correction to account for the contribution of large (infrequent) flares, and this correction is $\sim 10-25 \%$, depending on wavelength, with larger corrections for shorter wavelengths. The basic scheme is the same as in Ribas et al. (2016), and the actual details are discussed for each wavelength interval below. Of course, future detailed multiwavelength studies of Proxima flares will be able to provide much better constraints.

\subsection{X-rays: $X M M$}

In the 0.7 to $3.8 \mathrm{~nm}$ range we used four XMM-Newton observations with IDs 0049350101, 0551120201, 0551120301, and 0551120401. The first dataset, with a duration of $67 \mathrm{ks}$, was studied by Güdel et al. (2004) and contains a very strong flare

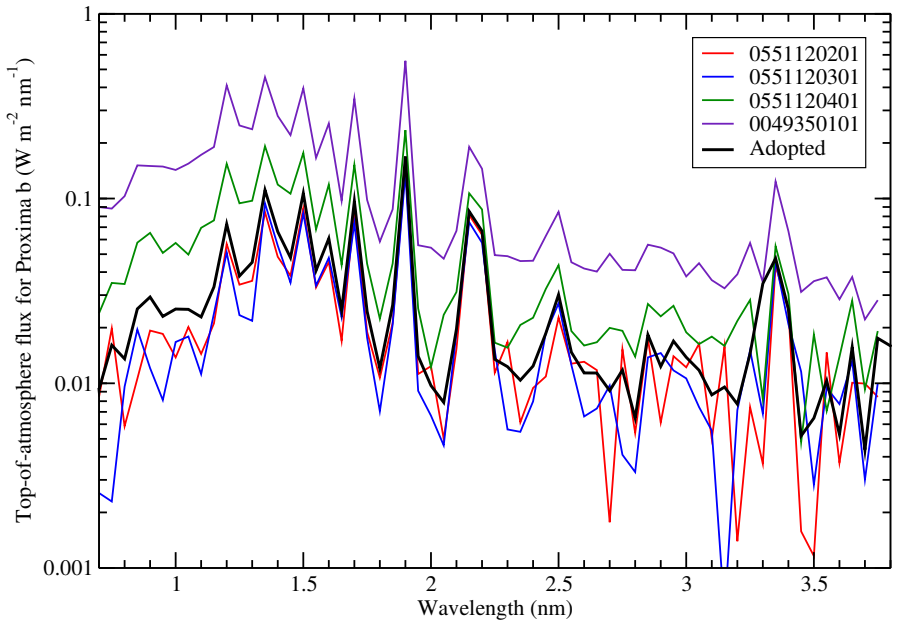

Fig. 1. Comparison of the top-of-atmosphere fluxes for Proxima b corresponding to $4 X M M$-Newton datasets (in color). The spectrum with higher fluxes (0049350101) corresponds to an observation that includes a strong flare event. Our adopted representative mean flux is depicted in black, and has been corrected to the mean point of the activity cycle and considers contributions from flares.

with a total energy of $\approx 2 \times 10^{32} \mathrm{erg}$. The other three datasets (adding to a total of $88 \mathrm{ks}$ ), were studied by Fuhrmeister et al. (2011), and include several flares, the strongest of which has an energy of about $2 \times 10^{31} \mathrm{erg}$. As in Ribas et al. (2016) we adopt the total spectrum corresponding to the combined 88-ks datasets and an additional energetic flare correction corresponding to a flux multiplicative scaling factor of 1.25. The comparison of the four individual observations with our adopted spectrum is shown in Fig. 1. The observation with the strong flare has fluxes that are three to five times higher than our average representative spectrum.

X-ray observations of Proxima were also obtained with other facilities, namely the Chandra observatory and the Swift mission. Chandra is optimized for high spatial and spectral resolution, which is not relevant to the determination of the SED of Proxima, and its flux calibration has larger uncertainty than that of XMM-Newton (Plucinsky et al. 2017). As for Swift, both its sensitivity and spectral resolution are significantly below that of XMM-Newton. Thus, including Chandra and Swift observations in our analysis would not contribute significantly to the quality of the derived SED but instead add complexity and potential for systematic errors. For these reasons, we prefer to base our hard $\mathrm{X}$-ray SED solely on XMM-Newton data.

A detailed analysis of Proxima X-ray observations obtained by Swift and other facilities was recently published by Wargelin et al. (2017) and they find good consistency between the different integrated X-ray flux measurements. Furthermore, the authors present evidence of a $\sim 7-y r$ activity cycle with an amplitude of $L_{\mathrm{X}}^{\mathrm{max}} / L_{\mathrm{X}}^{\mathrm{min}} \approx 1.5$ and note that the XMM observations (which are the same we use) correspond to X-ray cycle maxima (years 2001 and 2009). This implies that a correction should be made to refer them to the cycle average. We did so by adopting a multiplicative factor of 0.83 applied to the fluxes to yield our final values.

\subsection{X-rays: ROSAT}

ROSAT observations were used in the wavelength range from 3.8 to $10 \mathrm{~nm}$. Four suitable datasets are available from the ROSAT archive, with IDs RP200502N00, RP200502A01, 
Table 1. List of facilities, instruments and methods employed to determine the full spectral energy distribution of Proxima.

\begin{tabular}{lllll}
\hline \hline$\lambda$ range $(\mathrm{nm})$ & Facility/Instrument & Dataset & Section & Method \\
\hline $0.7-3.8$ & XMM-Newton/RGS & 0551120201, 0551120301, 0551120401 & 2.1 & Combined spectrum, energetic flare correction \\
$3.8-10$ & ROSAT/PSPC & RP200502N00, RP200502A01, RP200502A02, RP200502A03 & 2.2 & Plasma fit, combined spectrum, energetic flare correction \\
$10-40$ & EUVE & proxima_cen_9305211911N & 2.3 & Spectrum, energetic flare correction \\
$40-92$ & Model & & 2.4 & Scaled from H Lyman $\alpha$ \\
$92-117$ & FUSE & D1220101000 & 2.5 & Spectrum, geocoronal correction \\
$117-121.4$ and & HST/STIS E140M & O5EO01010, O5EO01020, O5EO01030, O5EO01040 & 2.6 & Combined spectrum \\
$121.7-170$ & & O5EO02010, O5EO02020, O5EO02040 & & \\
$121.4-121.7$ & HST/STIS E140M & O5EO01010, O5EO01020, O5EO01030, O5EO01040 & Fit to H Lyman $\alpha$ wings to correct for ISM absorption \\
$170-1000$ & HST/STIS G230LB, OCR7QQANQ, OCR7QQAOQ, OCR7QQARQ, OCR7QQASQ, & 2.7 & Spectrum \\
$1000-30000$ & G430L,G750L & OCR7QQAMQ, OCR7QQAPQ, OCR7QQAQQ & & \\
& Model & IR photometry (Table 4) & & Fit using BT-Settl models \\
\hline
\end{tabular}

RP200502A02, and RP200502A03. Their integration times were $3.8,7.9,20.3$, and $3.8 \mathrm{ks}$, and the observation dates 1992.3, 1993.2, 1993.7, and 1994.2, respectively. The analysis procedure is explained in Ribas et al. (2016). We calculated the average spectrum by using the integration time as the weight factor, and this should correspond a mean date of 1993.5, which is quite close to the midpoint of the activity cycle according to Wargelin et al. (2017). Comparison with the overlapping wavelength region with the XMM data indicates very good mutual agreement. A multiplicative scaling factor of 1.25 was further applied to include the energetic flare correction also in accordance with the procedure followed for XMM.

\subsection{EUV: EUVE}

For the extreme-UV range, covering from 10 to $40 \mathrm{~nm}$, we used the EUVE spectrum available from the mission archive with Data ID proxima_cen_9305211911N, corresponding to an integration time of $77 \mathrm{ks}$ and observation date 1993.5. The details of this observation are given in Ribas et al. (2016) and Linsky et al. (2014). We corrected this spectrum using a multiplicative scaling factor 1.25 to account for the average flux contribution coming from energetic flares. No activity cycle correction was necessary because the observation is close to the actual mid point (Wargelin et al. 2017).

\subsection{EUV: Lyman continuum}

The interval between 40 and $92 \mathrm{~nm}$ (Lyman limit) cannot be observed from Earth due to the very strong interstellar medium absorption, even for a star as nearby as Proxima. To estimate the flux in this wavelength range we make use of the theoretical calculations presented by Linsky et al. (2014). We adopt the model corresponding to intermediate activity (1303) because it best reproduces the H Ly $\alpha$ flux at the stellar surface (see Sect. 2.6). We consider the wavelength intervals $40-50 \mathrm{~nm}, 50-60 \mathrm{~nm}, 60$ $70 \mathrm{~nm}, 70-80 \mathrm{~nm}$, and $80-91 \mathrm{~nm}$, and the resulting ratios of the fluxes to the integrated H Ly $\alpha$ flux are $0.01,0.04,0.03,0.05$, and 0.12 , respectively. In our combined spectrum we consider these wavelength bins, yielding the appropriate integrated flux values. We note that the flux in this interval had been underestimated by about a factor of two in our previous calculations in Ribas et al. (2016).

\subsection{FUV: FUSE}

Data from FUSE were used to obtain the flux in part of the farUV range, from 92 to $117 \mathrm{~nm}$. We employed the spectrum with
Table 2. Ratios between the different H Lyman features using an intermediate activity model (1303) from Linsky et al. (2014).

\begin{tabular}{lrc}
\hline \hline Feature & Wavelength $(\mathrm{nm})$ & Ratio to H Ly $\alpha$ \\
\hline H Ly $\beta$ & 102.57 & 0.0195 \\
H Ly $\gamma$ & 97.25 & 0.0089 \\
H Ly $\delta$ & 94.97 & 0.0057 \\
H Ly $\epsilon$ & 93.78 & 0.0037 \\
H Ly 7 & 93.08 & 0.0025 \\
H Ly 8 & 92.62 & 0.0017 \\
H Ly 9 & 92.31 & 0.0012 \\
H Ly 10 & 92.10 & 0.0007 \\
H Ly 11+rest & $91.2-91.9$ & 0.0045 \\
\hline
\end{tabular}

Data ID D1220101000 with a total integration time of $45 \mathrm{ks}$ and observation date 2003.3 (another FUSE dataset exists, namely P1860701000, but it has much shorter duration - $6 \mathrm{ks}-$ and correspondingly lower signal-to-noise ratio). All obvious spectral regions with geocoronal emission were removed and only the wavelength intervals with stellar features (using the spectrum in Redfield et al. 2002, as a reference) were kept. The actual intervals are: $97.4-98 \mathrm{~nm}, 99.1-101.1 \mathrm{~nm}, 103.0-103.4 \mathrm{~nm}, 103.7-$ $103.8 \mathrm{~nm}, 110.9-113 \mathrm{~nm}$. These wavelength ranges include most of the features from stellar origin (notably three strong lines corresponding to $\mathrm{C}$ III and $\mathrm{O}$ VI, which account for $80 \%$ of the $92-$ $117 \mathrm{~nm}$ flux except for the H Lyman series) and no geocoronal contamination. These intervals are missing the flux from the $\mathrm{H}$ Lyman series from H Ly $\beta$ to the H Lyman limit and this needs to be considered.

We calculated the ratios between the different $\mathrm{H}$ Lyman features using an intermediate activity model (1303) from Linsky et al. (2014). The values are shown in Table 2. To produce a spectrum, we assumed the line profile from the H Ly $\alpha$ feature (see Sect. 2.6). For each of the H Lyman series lines we scaled the width to match the typical width of the stellar features (C III and $\mathrm{O}$ VI) and also the height of the emission to match the integrated flux. The results that we obtain are consistent with those presented by Guinan et al. (2003) and Ribas et al. (2005) for a Sun-like star with similar scaled H Lyman $\alpha$ flux.

Christian et al. (2004) found three flare events in the FUSE dataset that we employed, which produce an increase of up to one order of magnitude in the instantaneous flux. The combined effect of such flares is about $20-30 \%$ relative to the quiescent emission, which appears to be reasonable given our X-ray estimates below. Also, the observation date is close to the mid point of the activity cycle (Wargelin et al. 2017). Thus, no further corrections were applied. 


\subsection{FUV: HST/STIS E140M}

A high-quality spectrum from the StarCAT catalog (Ayres 2010) obtained with the HST Space Telescope Imaging Spectrograph (STIS; Woodgate et al. 1998) was used to measure the fluxes between 117 and $170 \mathrm{~nm}$ (except for $\mathrm{H} \operatorname{Ly} \alpha$ ). The spectrum was produced by co-adding a number of individual observations corresponding to HST datasets O5EO01010, O5EO01020, O5EO01030, O5EO01040, O5EO02010, O5EO02020, and O5EO02040 and with a total integration time of $35.7 \mathrm{ks}$ and observation date 2000.4. A flare analysis of these individual datasets was carried out by Loyd \& France (2014), who identified a number of flare events in the stronger emission lines. These flares contribute some $25-40 \%$ of the integrated flux (Loyd, priv. comm.) and thus represent similar values to those found in X-rays. In addition, as before, the date of the observations is nearly at the mid point of the activity cycle (Wargelin et al. 2017) and no further corrections were made. The intrinsic line profile of the $\mathrm{H} \mathrm{Ly} \alpha$ feature that we adopt was calculated from the same base spectrum by Wood et al. (2005). The relative flare contribution corrected for ISM absorption is estimated to be of $\sim 10 \%$ (Loyd, priv. comm.).

\subsection{UV to NIR: HST/STIS and HST/FOS}

Proxima was observed with HST/STIS on 24 April, 2015 as part of the Cycle 22 incarnation of the Next Generation Spectral Library (NGSL). The specific dataset references are listed in Table 1. The NGSL is an HST/STIS snapshot program which has compiled a spectral library of 570 representative spectral type stars for use in spectral synthesis of galaxies and other composite stellar systems. The spectra are obtained using the three low dispersion CCD modes of STIS, G230LB, G430L, and G750L, covering $\lambda \lambda 170-1020 \mathrm{~nm}$ at a resolution of about 1000 . For Proxima, the exposure times were $2 \times 600 \mathrm{~s}, 2 \times 30 \mathrm{~s}$, and $30 \mathrm{~s}$ for the three gratings.

The G230LB and G430L spectra were obtained through the $0 \prime 2$ E1 aperture, located near one edge of the CCD to reduce charge transfer losses during readout. The G750L spectrum, also observed through a 0 !'2 slit, was obtained at the regular long slit center near the middle of the CCD. This was in order to take advantage of the very narrow 0'.09 slit during contemporaneous CCD fringe flat calibration exposures to improve the removal of the considerable (10-15\%) fringing above $700 \mathrm{~nm}$ in the G750L data.

To save valuable on-target time during the HST snapshots, no contemporaneous wavelength calibrations (WAVECALS) are carried out during NGSL observations. Instead, a generic wavelength calibration is supplied in the pipeline download of the data, and a linear zeropoint pixel shift is determined either from inspection or cross-correlation of a preliminary 1D extraction of the source with a velocity template spectrum. This pixel shift is then inserted into the FITS header of the 2D STIS data; subsequent extraction of $1 \mathrm{D}$ spectra using the task X1D in the STSDAS package of IRAF takes out the first-order grating setting difference between the actual observation and the generic wavelength solution, typically $3-5$ pixels.

The 1D spectra were extracted in IRAF/PYRAF using the X1D task. During extraction, the X1D task also applies charge transfer inefficiency corrections, corrects for slit losses in the 0.2 slit, and applies an overall flux calibration to units of $F_{\lambda}$. The G750L spectrum was defringed using the contemporaneous fringe flat obtained through the narrower slit which mimics a point source on the detector better than obtaining a flat through the 0 '.2 slit.
Table 3. Optical $U B V R I$ photometry of Proxima.

\begin{tabular}{cccccl}
\hline \hline$U$ & \multicolumn{1}{c}{$B$} & \multicolumn{1}{c}{$V$} & \multicolumn{1}{c}{$R$} & \multicolumn{1}{c}{$I$} & Ref. \\
\hline 14.55 & 13.12 & 11.22 & $8.970^{a}$ & $7.310^{a}$ & Mould \& Hyland (1976) \\
14.56 & 13.02 & 11.04 & $8.68^{a}$ & $6.42^{a}$ & Frogel et al. (1972) \\
& 12.988 & 11.11 & 9.429 & 7.442 & Reid (1982) \\
14.21 & 12.95 & 11.13 & 9.45 & 7.41 & Jao et al. (2014) \\
& 13.02 & 11.05 & & & Gliese \& Jahreiss (2015) \\
& & 11.05 & 9.43 & 7.43 & Bessell (1991) \\
\hline 14.56 & 12.988 & 11.11 & 9.429 & 7.442 & Adopted \\
\hline 14.492 & 13.000 & 11.147 & 9.399 & 7.374 & STIS synthetic \\
14.491 & 12.970 & 11.083 & 9.380 & 7.384 & STIS+FOS synthetic \\
\hline
\end{tabular}

Notes. Magnitudes are in the Johnson-Cousins system unless otherwise noted. ${ }^{(a)}$ Not in the Cousins photometric system. No reliable transformation to Cousins for such red object is available.

The G230LB mode of STIS suffers from contamination by scattered zero-order light from all wavelengths to which the detector is sensitive. This is corrected for using the procedure developed by Lindler \& Heap (2008). Briefly, the initial combined flux calibrated spectrum is run in reverse through the G230LB sensitivity function covering all wavelengths to produce a best estimate of the G230LB counts over the entire optical range. From this, a pixel-by-pixel correction is calculated from a simple wavelength-dependent function dependent on the total counts in the overall computed spectrum. This correction is then subtracted pixel-by-pixel from the G230LB counts spectrum to correct for the red light contamination of the UV spectrum, and then the G230LB counts spectrum is again flux calibrated.

Inspection of the modest wavelength overlap $(\sim 200 \mathrm{~nm})$ between the three low dispersion spectra shows that the absolute calibrations of three individual gratings agree to better than $2-3 \%$ for the observation of Proxima. The three individual grating spectra were combined into a single spectrum using the SCOMBINE and DISPCOR tasks in IRAF. The final spectrum covers 170 to $1020 \mathrm{~nm}$ with a constant sampling $0.2 \mathrm{~nm}$ per pixel.

\subsubsection{Comparison to ground-based photometry}

Standard $U B V R I$ photometry of Proxima was collected from the literature. Measurements from different sources are provided in Table 3. In the case of the $U$ band, one of the measurements is very discrepant from the other two. It is possible that the value from Jao et al. (2014), which is brighter by $0.3 \mathrm{mag}$ and corresponds to a single epoch, was affected by a flare. In contrast, the photometric measurements of Frogel et al. (1972) correspond to the average of several observations taken outside of flare activity and thus we adopt the $U$-band magnitude from this study. The quoted uncertainty is $0.05 \mathrm{mag}$. For the $B V R I$ bands we adopt the photometry from Reid (1982), which is the average of 24 individual measurements. The quoted uncertainty is $0.028 \mathrm{mag}$, although it is not certain to which band this value corresponds.

To compare the final STIS spectrum with the ground-based photometry, we calculated synthetic Johnson/Cousins photometric indices by convolving the STIS spectrum with $U B V R I$ bandpasses from Bessell \& Murphy (2012), with zero points calibrated via the STIS_008 Vega spectrum from the CALSPEC Calibration database ${ }^{1}$. The calculated magnitudes are listed in Table 3 . All bands agree with the adopted, best-reliable groundbased photometry of Proxima within 3-6\%.

\footnotetext{
l http://www.stsci.edu/hst/observatory/crds/calspec. html
} 


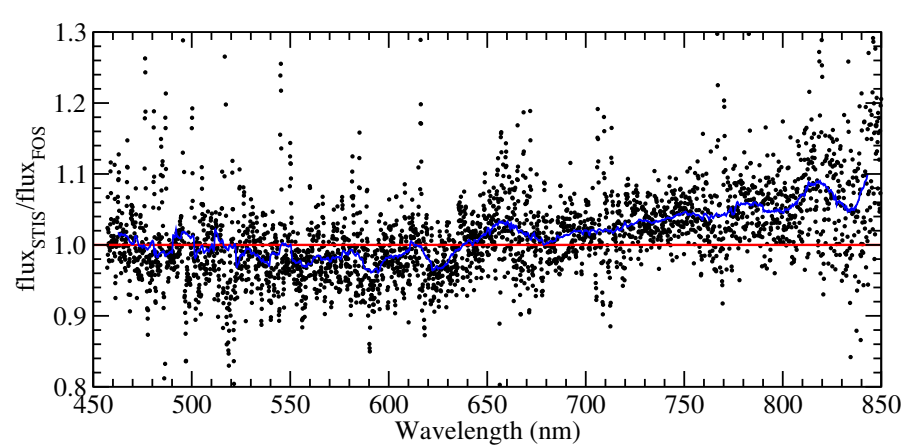

Fig. 2. Ratio of STIS to FOS fluxes shown as black circles for each wavelength bin. The blue line shows a 100-point running average.

\subsubsection{Comparison to HST/FOS}

HST observed Proxima with the Faint Object Spectrograph (FOS) on 1 July, 1996 through the $11^{\prime \prime} 0$ aperture for $430 \mathrm{~s}$ with the G570H grating (dataset Y2WY0305T) and 280 s with the G780 grating (dataset Y2WY0705T). The spectrum covers $\lambda \lambda 450$ $850 \mathrm{~nm}$ with a resolution of $0.09 \mathrm{~nm}$ and we compare the STIS and combined FOS spectra in Fig. 2. There is general agreement at the $\sim 5 \%$ level and thus the STIS spectrum agrees with the FOS data of Proxima at a similar level to the broad-band photometry.

\subsubsection{Final spectrum}

The FOS spectrum covers a subrange of the STIS spectrum and it does so at a higher spectral resolution. One could thus consider adopting a final spectrum composed of three wavelength intervals: 170-460 nm (STIS), 460-840 nm (FOS), 840-1000 nm (STIS). We have calculated the comparison with broad-band photometric measurements and this is shown in the last row of Table 3. As expected, the differences are rather minor with the all-STIS spectrum. Also, for most applications requiring irradiance measurements, the increased resolution in the central part of the optical wavelength range is of little use. Given these considerations, the results of the comparison between the STIS and FOS fluxes, and the interest of preserving homogeneity, we decided to adopt the full wavelength coverage from STIS as a fair representation of the spectral energy distribution of Proxima over the wavelength region of comparison.

As occurs at high energies, Proxima is also known to experience flux variations in the optical due to the presence of surface inhomogeneities (Anglada-Escudé et al. 2016; Wargelin et al. 2017). Photometric monitoring of Proxima shows that the peakto-peak variability with respect to the mean is of the order of $5 \%$ in the $B$ band and $2 \%$ in the $V$ band over timescales of months, and can be attributed to rotational modulation. This provides a viable explanation for the 3-6\% difference in the results of the comparisons between different measurements. The analysis of Davenport et al. (2016) using MOST satellite observations covering roughly 430 to $760 \mathrm{~nm}$ and taken over a time period of nearly 38 days reveal frequent white-light flares. There are five to eight measurable flares per day with a typical duration of $\sim 1 \mathrm{~h}$. However, the average flux contribution from flares in this wavelength range to the quiescent flux is only $2.6 \%$ (Davenport, priv. comm.). This relatively small effect, less than the typical uncertainty of the absolute flux calibration, suggests that flare correction to optical (and IR) spectrophotometric and photometric observations is not necessary. The flux values for Proxima that we provide should be representative of the average flux to better than $5 \%$.

\subsection{IR: model spectrum}

As we have shown, spectrophotometric observations that can be calibrated to yield physical fluxes are available for most wavelength regions up to about $1 \mu \mathrm{m}$. Beyond this wavelength value, the measurements are in the form of broad-band magnitudes or fluxes. We performed a search in the literature for flux measurements of Proxima. An important source of measurements is the catalog of Gezari et al. (1999), and we complemented it with subsequent references. A summary of the photometry is given in Table 4 . In view of the uncertainties and absolute calibration of the photometric systems, we decided to adopt two independent photometric datasets, namely the 2MASS $J H K$ photometry (although the 2MASS $K$ band measurement has a flag indicating poor quality) and the Mould \& Hyland (1976) JHKL photometry, which has two epochs and is given in a well-calibrated standard system. No $M$-band photometry was used in view of the large uncertainty. The magnitudes were transformed into physical flux units using the zero-point calibrations in Cohen et al. (2003) for the 2MASS system and Bessell et al. (1998) for the photometry from Mould \& Hyland (1976). The fluxes are given in Table 5. We additionally considered the recent revision of the zero points for the near-infrared (NIR) magnitudes by Mann \& von Braun (2015) but the results are very similar.

Besides the photometry in the classical broad-band systems, flux measurements of Proxima coming a number of space missions also exist, namely WISE, MSX, IRAS, and Spitzer. For the WISE mission (Wright et al. 2010) we considered both the AllWISE and the WISE All-Sky Source catalogs. In both cases, the $W 1$ and $W 2$ magnitudes are saturated (17\% to $25 \%$ saturated pixels), while the $W 3$ and $W 4$ bands are not. The agreement for the $W 1, W 3$ and $W 4$ bands for both catalogs is good but the $W 2$ magnitudes are highly discrepant. The $W 2$ magnitude from the WISE All-Sky Source catalog leads to an unphysical energy distribution (much higher flux than in all other bands). Also, the uncertainties associated to the saturated bands of the WISE All-Sky Source catalog seem unrealistically low. We decided to adopt the AllWISE measurements and uncertainties but did not consider the saturated $W 1$ an $W 2$ bands in the fits. The physical fluxes for the WISE bands were calculated using zero points in Jarrett et al. (2011) and are listed in Table 5. Proxima is included in the MSX6C Infrared Point Source Catalog (Egan et al. 2003). Measurements are only available in the so-called $A$ and $C$ bands and are given in physical units. These are included in Table 5. Proxima was also observed by the IRAS mission in two bands and has an entry in the IRAS catalog (Helou \& Walker 1988), with measurements in two bands $(12 \mu \mathrm{m}$ and $25 \mu \mathrm{m})$. The fluxes are provided in physical units and are listed in Table 5. Finally, Gautier et al. (2007) included Proxima in their survey of the farIR properties of $\mathrm{M}$ dwarfs and obtained a flux measurement in the Spitzer/MIPS 24- $\mu \mathrm{m}$ band. This is listed in Table 5. In addition to the bands considered above, we also included an anchor point from the HST/STIS G750L calibrated spectrophotometry at a wavelength of $1 \mu \mathrm{m}$, taking advantage of the very precise flux calibration of HST/STIS and to tie in with the optical measurements. For this, we considered an ad hoc square passband of $40 \mathrm{~nm}$ in width and calculated the average flux in this wavelength interval.

The fluxes in Table 5 are quite consistent for all bands except for the measurements of WISE W3 and IRAS_12, which correspond to nearly identical effective wavelengths but differ by over $50 \%$. While we initially employed the passband zero points from the literature, we explored another approach, namely the 
Table 4. Infrared JHKLM photometry of Proxima.

\begin{tabular}{llllll}
\hline \hline \multicolumn{1}{c}{$J$} & \multicolumn{1}{c}{$H$} & \multicolumn{1}{c}{$K$} & \multicolumn{1}{c}{$L$} & $M$ & Ref. \\
\hline 5.34 & 4.71 & 4.36 & & & Bessell (1991; Glass system) \\
& $4.73 \pm 0.05$ & $4.40 \pm 0.05$ & $4.17 \pm 0.01$ & & Frogel et al. (1972; SAO system) \\
& & $4.6 \pm 0.1$ & $4.1 \pm 0.1$ & $4.0 \pm 0.2$ & Frogel et al. (1972; Minnesota system) \\
$5.39 \pm 0.03$ & $4.74 \pm 0.02$ & $4.38 \pm 0.02$ & 4.15 & Aumann \& Probst (1991; CTIO system) \\
$\mathbf{5 . 3 5 7} \pm \mathbf{0 . 0 2 3}$ & $\mathbf{4 . 8 3 5} \pm \mathbf{0 . 0 5 7}$ & $\mathbf{4 . 3 8 4} \pm \mathbf{0 . 0 3 3}$ & & Cutri et al. (2003; 2MASS) \\
& $4.73 \pm 0.1$ & $4.40 \pm 0.1$ & & Veeder (1974; Johnson system) \\
$\mathbf{5 . 3 3 0} \pm \mathbf{0 . 0 2 0}$ & $\mathbf{4 . 7 2 5} \pm \mathbf{0 . 0 2 0}$ & $\mathbf{4 . 3 6 5} \pm \mathbf{0 . 0 2 8}$ & $\mathbf{4 . 0 4} \pm \mathbf{0 . 0 6}$ & Mould \& Hyland (1976; Glass system) \\
\hline
\end{tabular}

Notes. The adopted values are highlighted in bold face. ${ }^{(a)}$ Saturated, flag E.

Table 5. Infrared fluxes of Proxima.

\begin{tabular}{|c|c|c|c|c|c|c|c|}
\hline Band & $\begin{array}{l}\lambda_{\mathrm{eff}} \\
(\mu \mathrm{m})\end{array}$ & Magnitude & Error & $\begin{array}{c}\text { Flux } \\
\left(\mathrm{erg} \mathrm{s}^{-1} \mathrm{~cm}^{-2} \AA^{-1}\right)\end{array}$ & $\begin{array}{c}\text { Error } \\
\left(\mathrm{erg} \mathrm{s}^{-1} \mathrm{~cm}^{-2} \AA^{-1}\right) \\
\end{array}$ & $\begin{array}{l}\text { Flux (STIS_008) } \\
\left(\mathrm{erg} \mathrm{s}^{-1} \mathrm{~cm}^{-2} \AA^{-1}\right)\end{array}$ & $\begin{array}{l}\text { Passband } \\
\text { source }\end{array}$ \\
\hline G750L & 1.00 & & & $2.37 \times 10^{-12}$ & $4.7 \times 10^{-14}$ & & Square $(F W H M=40 \mathrm{~nm})$ \\
\hline$J$ (2MASS) & 1.24 & 5.357 & 0.023 & $2.25 \times 10^{-12}$ & $5.6 \times 10^{-14}$ & $2.25 \times 10^{-12}$ & Cutri et al. (2003) \\
\hline$H$ (2MASS) & 1.65 & 4.835 & 0.057 & $1.32 \times 10^{-12}$ & $7.9 \times 10^{-14}$ & $1.33 \times 10^{-12}$ & Cutri et al. (2003) \\
\hline$K$ (2MASS) & 2.16 & 4.384 & 0.033 & $7.55 \times 10^{-13}$ & $2.7 \times 10^{-14}$ & $7.55 \times 10^{-13}$ & Cutri et al. (2003) \\
\hline$J(\mathrm{MH76})$ & 1.24 & 5.330 & 0.020 & $2.32 \times 10^{-12}$ & $4.7 \times 10^{-14}$ & $2.34 \times 10^{-12}$ & Bessell et al. (1998) \\
\hline$H(\mathrm{MH76})$ & 1.64 & 4.725 & 0.020 & $1.45 \times 10^{-12}$ & $2.9 \times 10^{-14}$ & $1.48 \times 10^{-12}$ & Bessell et al. (1998) \\
\hline$K(\mathrm{MH} 76)$ & 2.19 & 4.365 & 0.028 & $7.11 \times 10^{-13}$ & $2.1 \times 10^{-14}$ & $7.17 \times 10^{-13}$ & Bessell et al. (1998) \\
\hline$L(\mathrm{MH} 76)$ & 3.50 & 4.04 & 0.06 & $1.71 \times 10^{-13}$ & $1.0 \times 10^{-14}$ & $1.74 \times 10^{-13}$ & Bessell et al. (1998) \\
\hline$W 1$ (AllWISE) $^{a}$ & 3.42 & 4.207 & 0.331 & $1.70 \times 10^{-13}$ & $5.9 \times 10^{-14}$ & $1.75 \times 10^{-13}$ & Wright et al. (2010) \\
\hline$W 2$ (AllWISE) $^{a}$ & 4.49 & 3.779 & 0.131 & $7.44 \times 10^{-14}$ & $1.0 \times 10^{-14}$ & $7.59 \times 10^{-14}$ & Wright et al. (2010) \\
\hline$W 3$ (AllWISE) $^{a}$ & 10.6 & 3.838 & 0.015 & $1.90 \times 10^{-15}$ & $3.8 \times 10^{-17}$ & $2.28 \times 10^{-15}$ & Wright et al. (2010) \\
\hline W4 (AllWISE) ${ }^{a}$ & 21.8 & 3.688 & 0.025 & $1.70 \times 10^{-16}$ & $4.2 \times 10^{-18}$ & $1.69 \times 10^{-16}$ & Wright et al. (2010) \\
\hline MSX $A$ & 8.38 & & & $8.00 \times 10^{-15}$ & $3.3 \times 10^{-16}$ & & Egan et al. (1999) \\
\hline MSX $C$ & 12.0 & & & $2.02 \times 10^{-15}$ & $1.8 \times 10^{-16}$ & & Egan et al. (1999) \\
\hline IRAS_12 & 10.4 & & & $3.18 \times 10^{-15}$ & $1.9 \times 10^{-16}$ & & Joint IRAS Science (1994) \\
\hline IRAS_25 & 21.8 & & & $1.19 \times 10^{-16}$ & $2.3 \times 10^{-17}$ & & Joint IRAS Science (1994) \\
\hline MIPS_24 & 23.3 & & & $1.30 \times 10^{-16}$ & $2.0 \times 10^{-19}$ & & Rieke et al. (2004) \\
\hline
\end{tabular}

Notes. ${ }^{(a)}$ Magnitudes from the WISE All-Sky Source catalog are $W 1=4.195 \pm 0.086, W 2=3.571 \pm 0.031, W 3=3.826 \pm 0.035, W 4=$ $3.664 \pm 0.024$.

calibration of the fluxes using a standard spectrum. As before, we used the STIS_008 Vega spectrum from the CALSPEC Calibration database. We calculated integrated fluxes for the relevant broad-band passbands using the definitions from the references in Table 4 and used them to set the zero point of the magnitude scale. The fluxes for Proxima calculated in this way are also listed in Table 5. As expected, the comparison between the literature zero points and those estimated using the spectrum of Vega reveals little differences in most cases (less than 3\%). However, the WISE W3 band zero point is notably different (by about $20 \%$ ) when comparing both methods. The value that we calculated from the Vega standard spectrum leads to closer agreement (though still far from perfect) with the IRAS_12 value. Given this circumstance, we decided to adopt the fluxes as calculated by us from the Vega spectrum for all bands with magnitude measurements (not for fluxes given in physical units).

Our procedure to obtain the NIR SED of Proxima is to fit all flux measurements with a spectrum from a theoretical model. As already mentioned in Sect. 2, no specific correction for flares was made. We chose to use the BT-Settl grid from Baraffe et al. $(2015)^{2}$. Proxima's surface gravity and metallicity are compatible within the error bars with values of $\log g=5.0$ and $[\mathrm{Fe} / \mathrm{H}]=0.0$ (Passegger et al. 2016), which are part of the BT-Settl model grid, and those were adopted in our SED fitting 2 In its latest version available from https://phoenix.ens-lyon.
$\mathrm{fr} /$ Grids/BT-Settl/CIFIST2011_2015/ procedure. The free parameters of the fit were the effective temperature and the angular diameter. For the latter, however, we used a prior from Demory et al. (2009) of $\theta=1.011 \pm 0.052$ mas. From the model spectra we calculated integrated fluxes for all passbands using the definitions from the references in Table 4. We built a $\chi^{2}$ statistic by comparing the model fluxes with the observed values and adopting the usual weight proportional to $1 / \sigma^{2}$, and this was minimized via the simplex algorithm as implemented by Press et al. (1992). To further constrain the model we doubled the weight of the anchor point at $1 \mu \mathrm{m}$ and of the angular diameter measurement.

Using the constraints above, the SED fit yields an effective temperature of $2870 \mathrm{~K}$ but an angular diameter that is $2.7 \sigma$ larger than the observation. Also, the residuals reveal a strong systematic difference between the bands roughly at either side of $3 \mu \mathrm{m}$. Such discrepancy suggests that Proxima has higher fluxes at longer wavelengths than expected from models. We then considered a fit only to the bands shortwards of $3 \mu \mathrm{m}$ and this led to an effective temperature of $3000 \mathrm{~K}$ and an angular diameter within $1 \sigma$ of the measured value. In Table 6 we list the flux residuals and resulting parameters from two fitting scenarios. We adopted the solution that fits the bands shorter than $3 \mu \mathrm{m}$, the HST/STIS flux and $J H K$ bands, our Fit 1. Figure 3 illustrates this fit and the comparison between models and observations. In addition, we ran tests by considering only measurements up to $2 \mu \mathrm{m}$, thereby excluding the $K$ band; these also show the same systematic trend and yielded very similar results. 
I. Ribas et al.: The full spectral radiative properties of Proxima Centauri

Table 6. SED model fits to Proxima IR flux measurements.

\begin{tabular}{|c|c|c|c|c|c|c|c|c|c|}
\hline \multirow[t]{2}{*}{ Band } & \multirow{2}{*}{$\begin{array}{c}\lambda_{\mathrm{eff}} \\
(\mu \mathrm{m})\end{array}$} & \multicolumn{4}{|c|}{ Fit 1} & \multicolumn{4}{|c|}{ Fit 2} \\
\hline & & Flux (mod) & obs-mod & $(\mathrm{obs}-\mathrm{mod}) / \sigma$ & used & Flux (mod) & obs-mod & $($ obs-mod $) / \sigma$ & used \\
\hline G750L & 1.000 & $2.43 \times 10^{-12}$ & $-5.8 \times 10^{-14}$ & -1.2 & $\mathrm{y}$ & $2.42 \times 10^{-12}$ & $-5.2 \times 10^{-14}$ & -1.1 & $\mathrm{y}$ \\
\hline$J$ (2MASS) & 1.245 & $2.26 \times 10^{-12}$ & $-1.4 \times 10^{-14}$ & -0.2 & $\mathrm{y}$ & $2.38 \times 10^{-12}$ & $-1.2 \times 10^{-13}$ & -2.2 & $\mathrm{y}$ \\
\hline$H(2 \mathrm{MASS})$ & 1.647 & $1.43 \times 10^{-12}$ & $-9.8 \times 10^{-14}$ & -1.2 & $\mathrm{y}$ & $1.50 \times 10^{-12}$ & $-1.7 \times 10^{-13}$ & -2.1 & $\mathrm{y}$ \\
\hline$K$ (2MASS) & 2.162 & $6.68 \times 10^{-13}$ & $8.7 \times 10^{-14}$ & 3.2 & $\mathrm{y}$ & $7.15 \times 10^{-13}$ & $4.0 \times 10^{-14}$ & 1.5 & $\mathrm{y}$ \\
\hline$J$ (MH76) & 1.240 & $2.26 \times 10^{-12}$ & $7.5 \times 10^{-14}$ & 1.6 & $\mathrm{y}$ & $2.37 \times 10^{-12}$ & $-3.4 \times 10^{-14}$ & -0.7 & $\mathrm{y}$ \\
\hline$H(\mathrm{MH} 76)$ & 1.642 & $1.42 \times 10^{-12}$ & $6.4 \times 10^{-14}$ & 2.1 & $\mathrm{y}$ & $1.48 \times 10^{-12}$ & $-4.3 \times 10^{-15}$ & -0.1 & $\mathrm{y}$ \\
\hline$K(\mathrm{MH76})$ & 2.195 & $6.28 \times 10^{-13}$ & $8.8 \times 10^{-14}$ & 4.2 & $\mathrm{y}$ & $6.72 \times 10^{-13}$ & $4.5 \times 10^{-14}$ & 2.1 & $\mathrm{y}$ \\
\hline$L(\mathrm{MH} 76)$ & 3.501 & $1.44 \times 10^{-13}$ & $3.0 \times 10^{-14}$ & 3.0 & $\mathrm{n}$ & $1.59 \times 10^{-13}$ & $1.5 \times 10^{-14}$ & 1.5 & $\mathrm{y}$ \\
\hline W1 (AllWISE) & 3.419 & $1.53 \times 10^{-13}$ & $2.2 \times 10^{-14}$ & 0.4 & $\mathrm{n}$ & $1.67 \times 10^{-13}$ & $7.9 \times 10^{-15}$ & 0.1 & $\mathrm{n}$ \\
\hline W2 (AllWISE) & 4.494 & $5.48 \times 10^{-14}$ & $2.1 \times 10^{-14}$ & 2.1 & $\mathrm{n}$ & $6.04 \times 10^{-14}$ & $1.5 \times 10^{-14}$ & 1.6 & $\mathrm{n}$ \\
\hline W3 (AllWISE) & 10.600 & $2.05 \times 10^{-15}$ & $2.3 \times 10^{-16}$ & 5.0 & $\mathrm{n}$ & $2.33 \times 10^{-15}$ & $5.0 \times 10^{-17}$ & -1.1 & $\mathrm{y}$ \\
\hline W4 (AllWISE) & 21.829 & $1.46 \times 10^{-16}$ & $2.3 \times 10^{-17}$ & 5.4 & $\mathrm{n}$ & $1.67 \times 10^{-16}$ & $2.5 \times 10^{-18}$ & 0.6 & $\mathrm{y}$ \\
\hline MSX $A$ & 8.382 & $6.07 \times 10^{-15}$ & $1.9 \times 10^{-15}$ & 5.8 & $\mathrm{n}$ & $6.84 \times 10^{-15}$ & $1.2 \times 10^{-15}$ & 3.5 & $\mathrm{y}$ \\
\hline MSX $C$ & 12.045 & $1.46 \times 10^{-15}$ & $5.6 \times 10^{-16}$ & 3.1 & $\mathrm{n}$ & $1.67 \times 10^{-15}$ & $3.5 \times 10^{-16}$ & 1.9 & $\mathrm{y}$ \\
\hline IRAS_12 & 10.375 & $2.35 \times 10^{-15}$ & $8.3 \times 10^{-16}$ & 4.4 & $\mathrm{n}$ & $2.68 \times 10^{-15}$ & $5.0 \times 10^{-16}$ & 2.6 & $\mathrm{y}$ \\
\hline IRAS_25 & 21.790 & $1.32 \times 10^{-16}$ & $-1.3 \times 10^{-17}$ & -0.6 & $\mathrm{n}$ & $1.50 \times 10^{-16}$ & $-3.1 \times 10^{-17}$ & -1.4 & $\mathrm{y}$ \\
\hline MIPS_24 & 23.245 & $1.14 \times 10^{-16}$ & $1.6 \times 10^{-17}$ & 80.7 & $\mathrm{n}$ & $1.30 \times 10^{-16}$ & $2.3 \times 10^{-19}$ & 1.2 & $\mathrm{y}$ \\
\hline
\end{tabular}

Notes. All fluxes are in units of erg s $\mathrm{s}^{-1} \mathrm{~cm}^{-2} \AA^{-1}$. The difference between Fit 1 and Fit 2 is the number of flux measurements used in the fitting procedure.

Table 7. Results from SED model fits to Proxima IR flux measurements.

\begin{tabular}{lcc}
\hline \hline Parameter & Fit 1 & Fit 2 \\
\hline$T_{\text {eff }}(\mathrm{K})$ & 3000 & 2870 \\
$\theta$ (mas) & 1.042 & 1.149 \\
$\chi_{v}^{2}$ & 3.48 & 8.22 \\
Flux $1-30 \mu \mathrm{m}$ & $2.33 \times 10^{-8}$ & $2.45 \times 10^{-8}$ \\
$\left(\operatorname{erg~s}^{-1} \mathrm{~cm}^{-2} \AA^{-1}\right)$ (calc.) & & \\
$\operatorname{Radius}^{a}\left(R_{\odot}\right)($ calc.) & 0.146 & 0.161 \\
\hline
\end{tabular}

Notes. ${ }^{(a)}$ Using the parallax measurement $\pi=0.7687 \pm 0.0003 \operatorname{arcsec}$ from Benedict et al. (1999).

\subsection{Combined spectrum}

The full spectrum, covering 0.7 to $30000 \mathrm{~nm}$, is provided in Table 8 and shown in Fig. 4 . Table 8 is available at the CDS and contains the following information: Col. 1 gives the wavelength in $\mathrm{nm}$ and Col. 2 lists the top-of-atmosphere flux for Proxima $b$ in units of $\mathrm{W} \mathrm{m}^{-2} \mathrm{~nm}^{-1}$, which are most commonly employed for planetary atmosphere work. The spectrum as obtained by adding the data from the various sources has rather inhomogeneous wavelength steps and was resampled using different bin sizes for different wavelength intervals depending on the quality of the spectrum. We calculated the top-of-atmosphere flux for Proxima $b$ by using the trigonometric distance to Proxima and by adopting an orbital distance of 0.0485 AU for Proxima b. Figure 4 also shows the top-of-atmosphere solar irradiance of the Earth for comparison, corresponding to the Thuillier et al. (2004) solar spectrum for medium solar activity. The integrated fluxes in various relevant intervals are listed in Table 9. Our results show that the XUV flux is nearly 60 times higher than Earth's value (0.0051 W m $\mathrm{W}^{-2}$; Ribas et al. 2016) and the total integrated flux is $877 \pm 44 \mathrm{~W} \mathrm{~m}^{-2}$, or $64 \pm 3 \%$ of the solar constant (top-of-atmosphere solar flux received by Earth, adopting $S_{\oplus}=1361 \mathrm{~W} \mathrm{~m}^{-2}$, Kopp \& Lean 2011). The adopted uncertainty on the total irradiance corresponds to a relative error of $5 \%$ on the Proxima flux (see below).

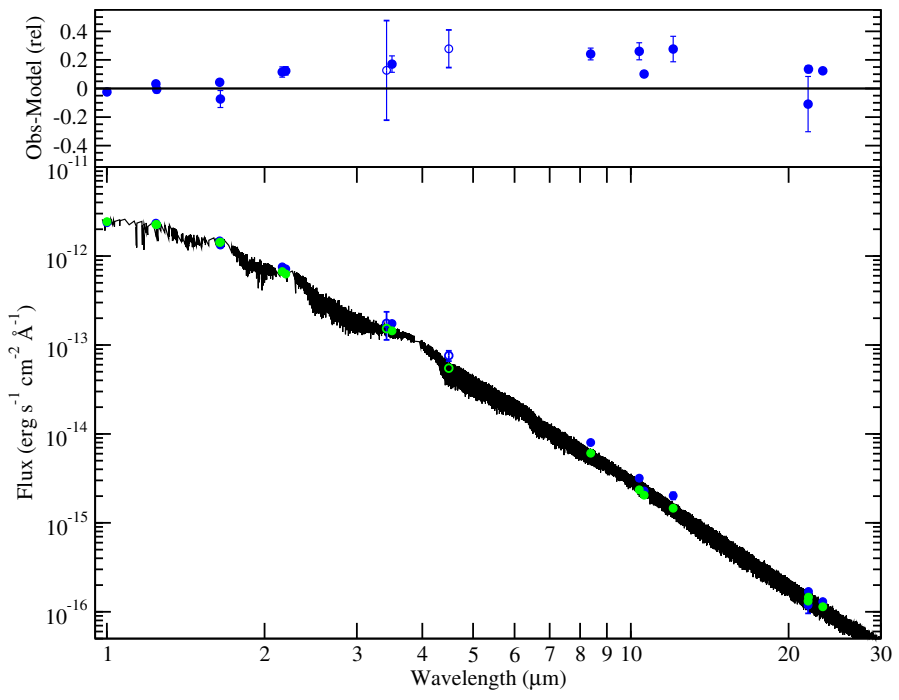

Fig. 3. Best-fitting model to IR fluxes considering measurements shortwards of $3 \mu \mathrm{m}$. The fit residuals and parameters are given in Tables 6 and 7 under the label Fit 1. The black line is the BT-Settl spectrum. The blue circles correspond to the observed fluxes and the green circles are the model-integrated fluxes. Empty symbols correspond to the WISE $W 1$ and $W 2$ bands, which are heavily saturated.

Proxima is variable over different timescales, most notably related to flare events (hours), rotational modulation (months) and activity cycle (years). In Table 9 we provide estimates of such variability amplitude (peak), when available, with respect to the mean flux value listed. Such estimates come from various literature sources that we have adapted to the relevant wavelength intervals. For the bolometric flux we scale the variability from that coming from the $V$ band. Wargelin et al. (2017) obtain a variation of $2 \%$ with respect to the average (4\% peak to peak). This, however, is not representative of the bolometric variability because activity-related effects are known to diminish with increasing wavelengths. In the case of Proxima, wavelengths around $1 \mu \mathrm{m}$ would be a better proxy for flux variations 
of the bolometric luminosity. We have used the StarSim simulator (Herrero et al. 2016) to estimate that variations of $2 \%$ in the $V$ band correspond to about $0.5 \%$ around $1 \mu \mathrm{m}$ if we assume spots with contrasts of 300-500 K (Berdyugina 2005). We adopt a similar scaling for the flare statistics obtained by Davenport et al. (2016), which correspond to the MOST satellite band. It is interesting to point out that the variability of the total irradiance of Proxima is about 25 times higher than the solar value $(0.02 \%$; Fröhlich 2012$)$ and this could have an impact on the climate forcing.

It should be noted that no information on the rotational modulation and cycle amplitude are available for the FUV range. The only relevant data in the UV comes from the results of Wargelin et al. (2017), who find a 4\% rotational modulation and similar cycle variability for the Swift/UVOT $W 1$ band, which has an effective wavelength of $260 \mathrm{~nm}$. Regarding flares, Walker (1981) studied several large events in the $U B V$ bands, and found peak-to-quiescence flux ratios of up to 25,4 and 1.5 , respectively. We do not include these values in Table 9 but we note that both the rotation/cycle amplitudes and the flare fluxes are strongly variable with wavelength.

\section{IR excess}

The flux residuals in Fig. 3 show a clear systematic offset beyond $\approx 2 \mu \mathrm{m}$, with the observed flux being $\sim 20 \%$ larger than model predictions. This systematic difference can be interpreted as a mid-to-near IR excess associated with the Proxima system, which, to our knowledge, has not been pointed out before. A possible physical explanation is the presence of dust grains, in what could be a warm ring close to the star, scattering the light from Proxima. The presence of such a dust reservoir could be leftover from the formation process of the planetary system around Proxima. Worth noting here is the K0 planet-host HD 69830 (Lovis et al. 2006), which was found to have a midIR excess ( $\sim 50 \%$ over photosphere $)$ and was interpreted by Beichman et al. (2011) as caused by small dust grains within 1 AU of the star. While no other warm disk around an old M dwarf has been reported, a cold resolved debris disk (an analog to the Kuiper Belt of our solar system) was found by Lestrade et al. (2012) with Herschel Space Observatory observations of GJ 581. Unfortunately, no far-IR measurements of Proxima are available to investigate the presence of a cold debris disk, which could lend additional support to the explanation of the mid-to-near IR excess that we find.

The systematic trend of the residuals could alternatively be related to certain shortcomings of the theoretical models. However, this is rather unlikely, as a $\sim 20 \%$ flux deficit is very significant and would have been identified before in other stars (Mann et al. 2015). In addition, one could think that the differences are related to the SED fitting procedure. A higher $T_{\text {eff }}$ value could yield fluxes in better agreement. Being in the Rayleigh-Jeans regime, this would mean a $\sim 20 \%$ increase in temperature of $\sim 600 \mathrm{~K}$. The other possibility is to assume a larger angular diameter by $\sim 10 \%$. Neither option can be valid because of the existence of strong constraints coming from the HST/STIS flux at $1 \mu \mathrm{m}$ and from the interferometric angular diameter determination. Finally, one could also consider a heavily spotted stellar surface (a hotter photosphere and a significant fraction of cooler spot areal coverage) that could result in a SED with an apparent IR flux excess. However, a IR flux excess that becomes significant at $\approx 2 \mu \mathrm{m}$ would require an unrealistically low spot temperature value $\left(T_{\text {spot }}<1500 \mathrm{~K}\right.$, $T_{\text {phot }}-T_{\text {spot }}>1500 \mathrm{~K}$; cf., Berdyugina 2005). Thus, given the lack of an alternative explanation consistent with the data and model fits, we find that the most likely cause of the IR excess is scattering of light from warm dust particles close to the star.

\section{Physical and radiative properties}

From the full spectral energy distribution of Proxima we can estimate its radiative parameters. The integration of the total flux (from X-rays to $30 \mu \mathrm{m}$ ) yields a flux of $2.86 \times 10^{-8} \mathrm{erg} \mathrm{s}^{-1} \mathrm{~cm}^{-2}$. The uncertainty of this value should be mostly driven by the uncertainty in the HST/STIS spectrophotometric measurements and of the IR fit. For the former, the absolute flux scale is found to be better than 5\%, and possibly better than $3 \%$ (Bohlin et al. 2014). For the IR, given the discussion on the quality of the fit, we also adopt an uncertainty of $5 \%$. Therefore, the bolometric flux of Proxima is found to be $F_{\text {bol }}=(2.86 \pm 0.14) \times$ $10^{-8} \mathrm{erg} \mathrm{s}^{-1} \mathrm{~cm}^{-2}$. This value and the angular diameter in Table 7 lead to an effective temperature value of $T_{\text {eff }}=2980 \pm 80 \mathrm{~K}$. The difference from the value in Table 7 arises because in this calculation we consider the full wavelength range, not only the interval beyond $1 \mu \mathrm{m}$. In other words, the optical flux of Proxima is lower than that of a $3000 \mathrm{~K}$ model and, hence, results in a lower $T_{\text {eff }}$.

Finally, we have calculated the bolometric luminosity by using the stellar parallax of Proxima from Benedict et al. (1999) and a solar luminosity value from IAU 2015 Resolution B3 on Recommended Nominal Conversion Constants for Selected Solar and Planetary Properties ${ }^{3}$. The bolometric luminosity of Proxima is, thus, $L_{\text {bol }}=(5.80 \pm 0.30) \times 10^{30} \mathrm{erg} \mathrm{s}^{-1}$ or $L_{\text {bol }}=0.00151 \pm 0.0008 L_{\odot}$.

Another SED for Proxima has been independently created by the MUSCLES Treasury Survey team to add to their cata$\log$ for $\mathrm{M}$ and $\mathrm{K}$ dwarf exoplanet host $\mathrm{stars}^{4}$, using data reduction procedures described in Loyd et al. (2016). In the discussion of their analysis, the authors mention some disagreements with the photometry and finally adopt a $T_{\text {eff }}$ of $2800 \mathrm{~K}$. The difference with our value most likely comes from the fact that we use a constraint provided by the observed angular diameter. Their bolometric flux is just above the $1 \sigma$ interval of the value we find.

\section{Time evolution of the flux received by Proxima $b$}

\subsection{Bolometric flux}

The total flux evolution of Proxima can be estimated from theoretical evolutionary model calculations. We employed the recent models of Baraffe et al. (2015) that are well suited for very low mass stars and include the most up-to-date physical ingredients. We linearly interpolated the evolutionary tracks from the models corresponding to 0.110 and $0.130 M_{\odot}$ to find a good match of the model predictions with our determined values for $L_{\mathrm{bol}}$ and $T_{\text {eff }}$ at the estimated age of the star of $4.8 \mathrm{Gyr}$ (Bazot et al. 2016). A stellar mass of $0.120 M_{\odot}$ yields the best simultaneous agreement of all parameters within the corresponding uncertainties, resulting in values of $L_{\mathrm{bol}}=0.00150 L_{\odot}$ and $T_{\text {eff }}=2980 \mathrm{~K}$. A formal uncertainty of $0.003 M_{\odot}$ can be estimated from the errors associated to $L_{\text {bol }}$ and $T_{\text {eff }}$. This is obviously a model-dependent estimate and no error in metallicity and $\log g$ was assumed. The evolutionary track of Proxima is shown in Fig. 5, in normalized

\footnotetext{
https://www .iau.org/administration/resolutions/ general_assemblies/

4 It can be found in https://archive.stsci.edu/prepds/ muscles/
} 


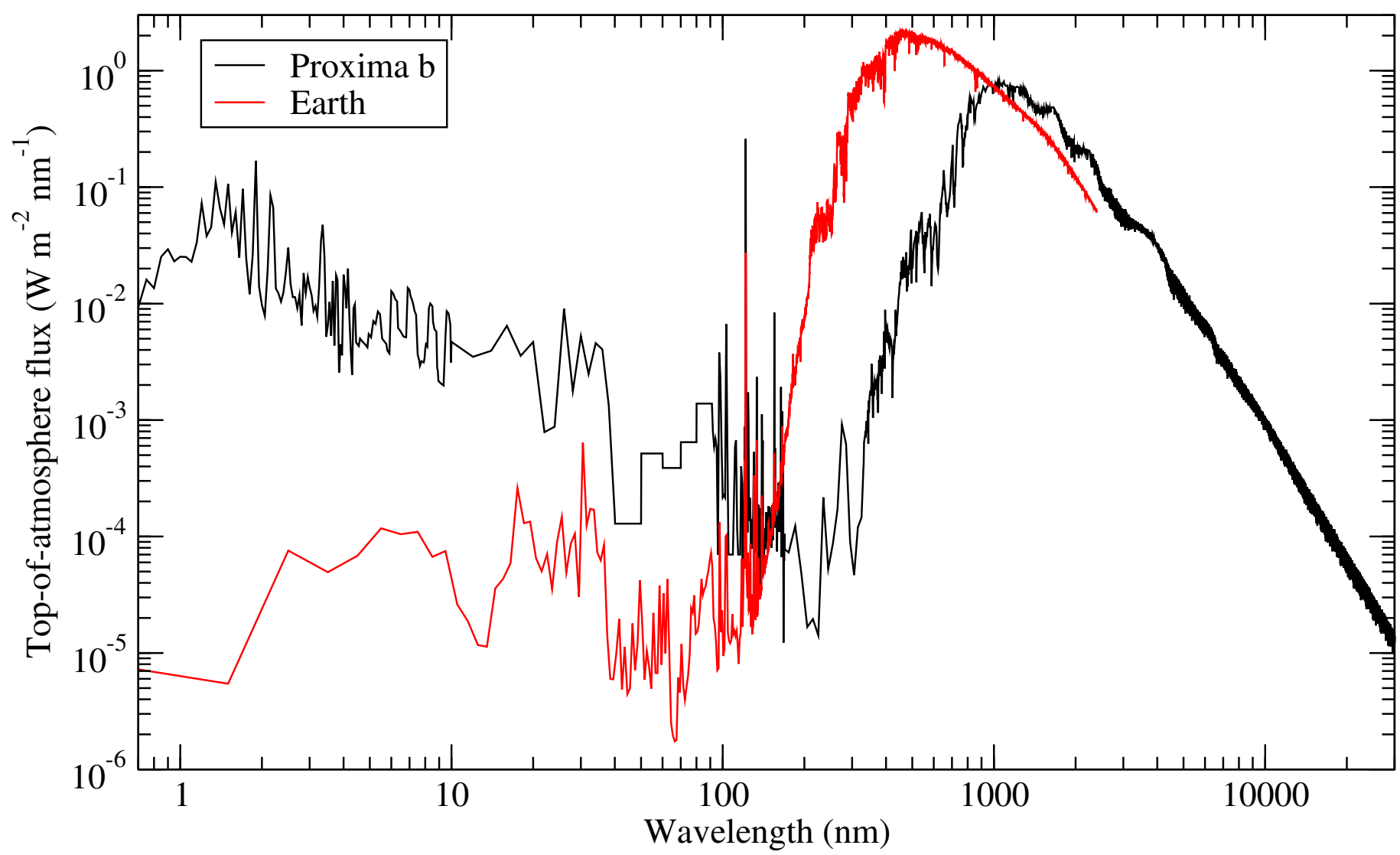

Fig. 4. Top-of-atmosphere full spectral irradiance received by Proxima b (black) and the Earth (red). An orbital distance of 0.0485 AU is assumed for Proxima $b$.

Table 9. Top-of-atmosphere (TOA) fluxes received currently by Proxima b.

\begin{tabular}{|c|c|c|c|}
\hline $\begin{array}{l}\text { Wavelength interval } \\
(\mathrm{nm})\end{array}$ & $\begin{array}{l}\text { TOA flux } \\
\left(\mathrm{W} \mathrm{m}^{-2}\right)\end{array}$ & Observed variability from mean & Ref. \\
\hline $0.7-10$ (X-rays) & 0.131 & $+100 \times($ flares $) ; \pm 20 \%$ (rotation) $\pm 20 \%$ (cycle) & 1,2 \\
\hline $10-40$ & 0.110 & $+100 \times($ flares $) ; \pm 20 \%$ (rotation) $; \pm 20 \%$ (cycle) & 1,2 \\
\hline $40-92$ & 0.033 & $+10 \times$ (flares); no rotation \& cycle information & 3 \\
\hline $92-118$ & 0.019 & $+30 \times$ (flares); no rotation \& cycle information & 4 \\
\hline $0.7-118$ (XUV) & 0.293 & $+100 \times($ flares $) ; \pm 20 \%$ (rotation) $; \pm 20 \%($ cycle $)$ & 1,2 \\
\hline $118-170$ (FUV) & 0.147 & $+10 \times$ (flares); no rotation \& cycle information & 5 \\
\hline $\mathrm{H} \operatorname{Ly} \alpha(122 \mathrm{~nm})$ & 0.130 & $+10 \times$ (flares); no rotation \& cycle information & 5 \\
\hline $0.7-30000\left(S_{\text {Proxb }}\right)$ & 877 & $+15 \%$ (flares); $\pm 0.5 \%$ (rotation); $\pm 0.5 \%$ (cycle) & 6,2 \\
\hline
\end{tabular}

Notes. Also provided are the peak variations with respect to the mean at various timescales related to stellar magnetic activity.

References. 1: Güdel et al. (2004); 2: Wargelin et al. (2017); 3: Mullan et al. (2006); 4: Christian et al. (2004); 5: Loyd \& France (2014), Loyd (priv. comm.); 6: Davenport et al. (2016).

units of today's bolometric luminosity. At $10 \mathrm{Myr}$, the time when the protoplanetary disk may have dissipated (Pecaut \& Mamajek 2016) and Proxima b became vulnerable to XUV radiation, the stellar luminosity was a factor of ten larger than today. Thus, Proxima b spent some 90-200 Myr in an orbit interior to the stellar habitable zone and possibly in a runaway greenhouse state. A detailed discussion is provided by Ribas et al. (2016).

\subsection{High-energy flux}

The evolution of the XUV flux of Proxima with time was addressed by Ribas et al. (2016). Here we revisit the calculations by considering also the early evolution of $L_{\mathrm{bol}}$ as the star was contracting towards the Main Sequence. The XUV evolution of young $\mathrm{M}$ dwarfs is poorly constrained but some tantalizing evidence exists indicating that the saturation limit of $\log \left(L_{\mathrm{X}} / L_{\text {bol }}\right) \approx-4$ also applies to the pre-Main Sequence (Preibisch \& Feigelson 2005). As we show above, the bolometric luminosity should have experienced significant changes over the first few hundred Myr in the history of Proxima and therefore this needs to be properly taken into account in the calculations (e.g., Luger \& Barnes 2015). Different XUV evolution laws are discussed in Ribas et al. (2016). One considers a saturated emission state up to a certain age followed by a power law decrease to today's XUV flux. The other one considers that Proxima has shown saturated behavior since its birth and until today. Observational evidence is still inconclusive as to which of these two XUV evolution scenarios is correct, and we hereby 


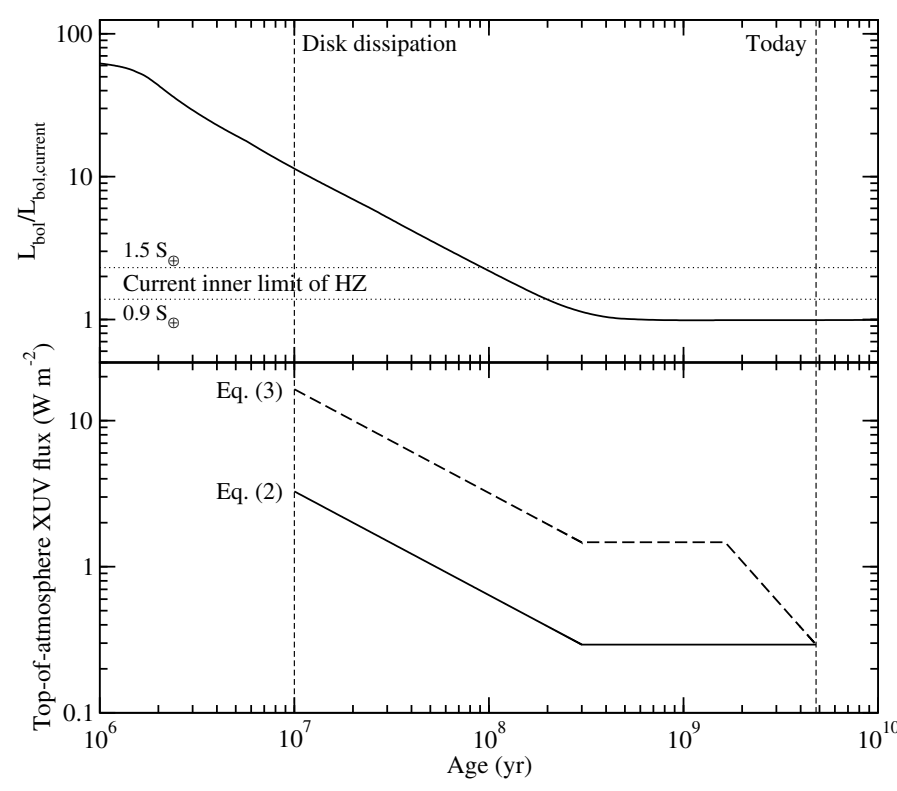

Fig. 5. Top: evolution of the bolometric luminosity of a $0.120-M_{\odot}$ star as predicted by the models of Baraffe et al. (2015) and normalized to today's Proxima luminosity $L_{\mathrm{bol}}=0.00151 L_{\odot}$. Marked with vertical dashed lines are today's Proxima age $(4.8 \mathrm{Gyr})$ and the approximate age at which the Proxima protoplanetary disk may have dissipated (10 Myr, Pecaut \& Mamajek 2016). The horizontal dotted lines mark the current inner limit of the habitable zone according to different assumptions on the spin rate of the planet (Kopparapu 2013): synchronous, that is, 1.5 times Earth's irradiance $\left(S_{\oplus}\right)$; asynchronous, that is, $0.9 S_{\oplus}$. Bottom: XUV flux evolution calculated using the two prescriptions explained in Sects. 5.2.1 (solid) and 5.2.2 (thick dashed). Vertical dashed lines mark the same features as in the top panel.

further consider them both. They should be representative of the extreme cases bracketing the real evolution of Proxima over its lifetime.

\subsubsection{Proxima is just at the end of the saturation phase}

Proxima's current relative X-ray value is $\log \left(L_{\mathrm{X}} / L_{\mathrm{bol}}\right)=-3.83$, which is very similar to the average of the distribution for stars between 0.1 and $0.2 M_{\odot}$ and ages of 0.1 to $10 \mathrm{Myr}$ found by Preibisch \& Feigelson (2005). This circumstance suggests that Proxima may still be today in the saturated regime and that $\log \left(L_{\mathrm{X}} / L_{\text {bol }}\right)=-3.83$ has been satisfied during its entire lifetime. This is in good agreement with the estimates of the saturation limit as determined from the equations in Reiners et al. (2014), which extends up to a rotation period of $P_{\text {rot }} \approx 80 \mathrm{~d}$ for a $0.146-R_{\odot}$ star, very close to Proxima's current rotation period of $P_{\text {rot }}=83 \mathrm{~d}$ (Suárez Mascareño et al. 2016).

To parameterize the bolometric flux, we consider the evolutionary model track and two different regimes, from 10 to $300 \mathrm{Myr}$ and from $300 \mathrm{Myr}$ to today. The evolution of the stellar bolometric flux as a function of the age $(\tau)$ in Myr can be approximated as (see top panel of Fig. 5):

$L_{\mathrm{bol}} / L_{\mathrm{bol}, \text { current }}=57.38 \tau^{-0.71}$ for $10 \mathrm{Myr}<\tau<300 \mathrm{Myr}$

$L_{\mathrm{bol}} / L_{\text {bol,current }}=1.000 \quad$ for $300 \mathrm{Myr}<\tau<4800 \mathrm{Myr}$. (1)

Then, we assume that the XUV flux scales in the same way as the X-rays. This is an approximation because the hardness ratio of the XUV spectrum may have softened as the star spun down. But in the absence of a better model, we used the evolution law from X-rays as valid for the full XUV range, and, therefore, that $\log \left(L_{\mathrm{XUV}} / L_{\mathrm{bol}}\right)=-3.48$ has remained constant for the entire lifetime of Proxima. With this, and the expressions in Eq. (1), we find the following relationship for the top-of-atmosphere flux of Proxima $b$ as a function of age (the current value is taken from Table 9):

$$
\begin{array}{ll}
F_{\text {XUV }}=16.81 \tau^{-0.71} \mathrm{~W} \mathrm{~m}^{-2} & \text { for } 10 \mathrm{Myr}<\tau<300 \mathrm{Myr} \\
F_{\mathrm{XUV}}=0.293 \mathrm{~W} \mathrm{~m}^{-2} & \text { for } 300 \mathrm{Myr}<\tau<4800 \mathrm{Myr} .
\end{array}
$$

The proposed evolution of the top-of-atmosphere flux received by Proxima $b$ corresponding to this scenario is illustrated in the bottom panel of Fig. 5 with a solid line.

\subsubsection{Proxima has evolved off saturation and is in the power law regime}

As an alternative to the XUV evolution scenario above, one can consider the results of Wright \& Drake (2016). Although based on 4 stars (among which is Proxima), the authors suggest that the X-ray evolution of fully convective stars is analogous to that of more massive Sun-like stars. In this case, to model the stage after saturation, we can adopt the relationship in Wright et al. (2011) by which $L_{\mathrm{X}} / L_{\mathrm{bol}} \propto R_{\circ}^{-2.70} \propto P_{\text {rot }}^{-2.70}$, where $R_{\circ} \equiv P_{\text {rot }} / \tau_{\mathrm{c}}$ is the so-called Rossby number (Noyes et al. 1984) and we assume the convective turnover time $\left(\tau_{\mathrm{c}}\right)$ to be constant during the main sequence lifetime of Proxima. From this, we can further adopt Mamajek \& Hillenbrand (2008), who find $P_{\text {rot }} \propto \tau^{0.566}$, where $\tau$ is the stellar age, to obtain $L_{\mathrm{X}} / L_{\mathrm{bol}} \propto \tau^{-1.5}$. Thus, considering that $\log \left(L_{\mathrm{X}} / L_{\mathrm{bol}}\right)=-3.83$ at an age of $4.8 \mathrm{Gyr}$ and that saturation of Sun-like stars occurs at an average value of $\log \left(L_{\mathrm{X}} / L_{\text {bol }}\right)=-3.13$ (Wright et al. 2011), we find that the end of saturation should have happened at an age of $1.64 \mathrm{Gyr}$.

As before, we further make the assumption that the total XUV flux follows the same evolution as the X-ray flux and we can write the relationship ( $\tau$ in Myr):

$$
\begin{array}{ll}
F_{\text {XUV }}=84.1 \tau^{-0.71} \mathrm{~W} \mathrm{~m}^{-2} & \text { for } 10 \mathrm{Myr}<\tau<300 \mathrm{Myr} \\
F_{\text {XUV }}=1.47 \mathrm{~W} \mathrm{~m}^{-2} & \text { for } 300 \mathrm{Myr}<\tau<1640 \mathrm{Myr} \\
F_{\text {XUV }}=9.74 \times 10^{4} \tau^{-1.5} \mathrm{~W} \mathrm{~m}^{-2} & \text { for } 1640 \mathrm{Myr}<\tau<4800 \mathrm{Myr} .
\end{array}
$$

This proposed evolution is illustrated in the bottom panel of Fig. 5 with a thick dashed line.

\subsection{XUV dose and water loss estimates}

The integration of the XUV relationships presented here and the comparison with the equivalent relationship for the Sun and the Earth (see Ribas et al. 2016) indicates that the total XUV dose that Proxima $\mathrm{b}$ has received over its lifetime is between 8 and 25 times greater than Earth's. But the most critical part may be the phase at which the atmosphere of Proxima $b$ was in runaway greenhouse effect, in an orbit interior to the habitable zone. The amount of XUV irradiation during this period of time from about 10 Myr until about 90-200 Myr could have caused an intense loss of water. To estimate the water loss, we proceeded as in Ribas et al. (2016) and Bolmont et al. (2017). We use the same units for the water loss as in those articles: $1 E O_{H}$ corresponds to the Earth ocean's worth of hydrogen. We also took into account the revised smaller mass for Proxima, but this has no significant impact on the calculations. With our model, we can estimate the current volatile losses of Proxima b: the hydrogen loss is of $0.003 E O_{H} / \mathrm{Myr}$, which corresponds 
Table 10. Hydrogen loss from Proxima $b$ for different XUV prescriptions.

\begin{tabular}{cccc}
\hline \hline XUV prescription & \multicolumn{3}{c}{$\mathrm{H}$ loss $\left(E O_{H}\right)$} \\
& $\begin{array}{c}\mathrm{HZ}\left(1.5 S_{\oplus}\right) \\
90 \mathrm{Myr}\end{array}$ & $\begin{array}{c}\mathrm{HZ}\left(0.9 S_{\oplus}\right) \\
200 \mathrm{Myr}\end{array}$ & $\begin{array}{c}\text { Lifetime } \\
4.8 \mathrm{Gyr}\end{array}$ \\
\hline Eq. (2) & 0.47 & 0.90 & 15.6 \\
Eq. (3) & 1.07 & 1.98 & 24.4 \\
\hline
\end{tabular}

Notes. The following assumptions are made: the initial time is $10 \mathrm{Myr}$, the mass and radius of Proxima $\mathrm{b}$ are $1.3 M_{\oplus}$ and $1.07 R_{\oplus}$, respectively, and the initial water content is considered infinite.

to $1.5 \times 10^{7} \mathrm{~g} \mathrm{~s}^{-1}$, the oxygen loss is $0.009 \mathrm{EO}_{H} / \mathrm{Myr}$, which corresponds to $4.3 \times 10^{7} \mathrm{~g} \mathrm{~s}^{-1}$.

Table 10 summarizes the results for the two prescriptions given in Eqs. (2) and (3). The parameterization of the XUV flux evolution given by Eq. (2) differs from the one used in Ribas et al. (2016) as follows: it is higher during the first $100 \mathrm{Myr}$ but lower by a factor $\sim 2.7$ during the following few Gyr. This has two consequences on the water loss: 1) during the runaway phase, and more especially during the first $100 \mathrm{Myr}$, the loss is more intense than in Ribas et al. (2016); and 2) on the long term, the total loss is lower. The parameterization of the evolution of the XUV flux given by Eq. (3) leads to higher XUV fluxes throughout the entire lifetime of Proxima $b$ when compared with Ribas et al. (2016).

If we assume synchronous rotation, our estimates indicate that Proxima b could have lost from $0.47 E O_{H}$ (Eq. (2)) to $1.07 \mathrm{EO}_{H}$ (Eq. (3)) between $10 \mathrm{Myr}$ and $90 \mathrm{Myr}$, when it reached the inner edge of the habitable zone at $1.5 S_{\oplus}$ (Kopparapu et al. 2016). Our new calculations therefore suggest that, during that time, Proxima b may have lost more water than previously estimated by Ribas et al. (2016), by about a factor 1.25 to 3. Assuming non-synchronous rotation, the amount of water lost could range from $0.9 \mathrm{EO}_{H}$ (Eq. (2)) to $1.91 \mathrm{EO}_{H}$ (Eq. (3)) between $10 \mathrm{Myr}$ and $200 \mathrm{Myr}$, when it reached the habitable zone inner edge at $0.9 S_{\oplus}$ (Kopparapu et al. 2014). The estimate obtained with the prescription of Eq. (2) is about the same value as previously provided by Ribas et al. (2016) while the calculation with Eq. (3) is about a factor of 2 larger.

In spite of the strong volatile losses $\left(\sim 0.5-2 E O_{H}\right)$, the planet could still have a significant amount of water reservoir when it entered the habitable zone depending on the initial content. What could have occurred beyond this point is quite uncertain. If we assume that the water loss processes were still active upon entering the habitable zone, we find that Proxima $b$ could have lost up to $15-25 E O_{H}$ during its lifetime. However, this needs to be considered an extreme upper limit because the volatile loss mechanisms would probably be significantly less efficient under such conditions (see discussion in Ribas et al. 2016).

\section{Conclusions}

This paper presents a full analysis of the SED of Proxima, covering X-rays to mid-IR, with the goal of providing useful input to study the atmosphere of Proxima $b$. We made use of measurements covering different wavelength intervals and acquired with various facilities (see Tables 1 and 5) to determine top-of-atmosphere fluxes from 0.7 to $30000 \mathrm{~nm}$, in steps of widths ranging from 0.05 to $10 \mathrm{~nm}$ depending on the wavelength range. Where spectrophotometric measurements were unavailable, we made use of theoretical models fitted using all available constraints. With the full spectral energy distribution and
Table 11. Summary of fundamental properties of Proxima.

\begin{tabular}{lc}
\hline \hline Parameter & Value \\
\hline$M\left(M_{\odot}\right)$ & $0.120 \pm 0.003^{a}$ \\
$R\left(R_{\odot}\right)$ & $0.146 \pm 0.007$ \\
$T_{\text {eff }}(\mathrm{K})$ & $2980 \pm 80$ \\
$F_{\text {bol }}\left(\mathrm{erg} \mathrm{s}^{-1} \mathrm{~cm}^{-2}\right)$ & $2.86 \pm 0.14 \times 10^{-8}$ \\
$L_{\text {bol }}\left(L_{\text {bol } \odot}\right)$ & $0.00151 \pm 0.00008$ \\
$\left\langle\log \left(L_{\mathrm{X}} / L_{\text {bol }}\right)\right\rangle$ & -3.83 \\
$\left\langle\log \left(L_{\mathrm{XUV}} / L_{\mathrm{bol}}\right)\right\rangle$ & -3.48 \\
$\mathrm{Age}(\mathrm{Gyr})^{b}$ & 4.8 \\
\hline
\end{tabular}

Notes. ${ }^{(a)}$ Model dependent, from errors in $T_{\text {eff }}$ and $L_{\text {bol }}$ but assuming no error in age and metallicity. ${ }^{(b)}$ From Bazot et al. (2016).

the available trigonometric distance, we could calculate the bolometric luminosity and the effective temperature. Also, Proxima has a quite accurate interferometric angular diameter measurement and this was used both to constrain the SED fit in the IR and to provide an empirical determination of the stellar radius. Interestingly, the fit of the IR SED revealed a flux excess $\approx 20 \%$ from Proxima. While the origin of this excess is uncertain, the most natural explanation is light scattering by dust particles in the Proxima system; additional observations can better ascertain the nature of the excess. The stellar mass was estimated by comparison with evolutionary models using the constraints provided by the radiative properties of Proxima. All the resulting fundamental parameters are summarized in Table 11.

Proxima is a benchmark star, not only for us to understand the stellar lower main sequence, but also, since the discovery of Proxima b, to study its habitable planet candidate. As discussed by Ribas et al. (2016), to determine the habitability of the planet it is essential to analyze the volatile loss processes that may affect its atmosphere, both currently and in the past. The detailed spectral energy distribution for Proxima presented here and the newly proposed XUV flux time evolution laws should help to provide the necessary constraints to model and interpret future observations of the nearest potentially habitable planet outside the solar system.

Acknowledgements. We are grateful to Rodrigo Luger for pointing out the increased XUV flux in the early evolution of Proxima, and to Parke Loyd for assistance with the flare characterization in the FUV range. We also gratefully acknowledge the insightful comments and suggestions by an anonymous referee. I.R. acknowledges support by the Spanish Ministry of Economy and Competitiveness (MINECO) and the Fondo Europeo de Desarrollo Regional (FEDER) through grant ESP2016-80435-C2-1-R, as well as the support of the Generalitat de Catalunya/CERCA programme. M.D.G. and T.S.B. acknowledge generous support provided by NASA through grant number GO-13776 from the Space Telescope Science Institute, which is operated by AURA, Inc., under NASA contract NAS5-26555. E.B. acknowledges funding by the European Research Council through ERC grant SPIRE 647383.

\section{References}

Anglada-Escudé, G., Amado, P. J., Barnes, J., et al. 2016, Nature, 536, 437 Aumann, H. H., \& Probst, R. G. 1991, ApJ, 368, 264

Ayres, T. R. 2010, ApJS, 187, 149

Baraffe, I., Homeier, D., Allard, F., \& Chabrier, G. 2015, A\&A, 577, A42 Barnes, R., Deitrick, R., Luger, R., et al. 2016, Astrobiology, submitted [arXiv: 1608.06919]

Bazot, M., Christensen-Dalsgaard, J., Gizon, L., \& Benomar, O. 2016, MNRAS, 460, 1254

Beichman, C. A., Lisse, C. M., Tanner, A. M., et al. 2011, ApJ, 743, 85

Benedict, G. F., McArthur, B., Chappell, D. W., et al. 1999, AJ, 118, 1086

Berdyugina, S. V. 2005, Living Rev. Solar Phys., 2, 8

Bessell, M. S. 1991, AJ, 101, 662

Bessell, M., \& Murphy, S. 2012, PASP, 124, 140

Bessell, M. S., Castelli, F., \& Plez, B. 1998, A\&A, 333, 231 
Bohlin, R. C., Gordon, K. D., \& Tremblay, P.-E. 2014, PASP, 126, 711 Bolmont, E., Selsis, F., Owen, J. E., et al. 2017, MNRAS, 464, 3728

Christian, D. J., Mathioudakis, M., Bloomfield, D. S., Dupuis, J., \& Keenan, F. P. 2004, ApJ, 612, 1140

Cohen, M., Wheaton, W. A., \& Megeath, S. T. 2003, AJ, 126, 1090

Cutri, R. M., Skrutskie, M. F., van Dyk, S., et al. 2003, VizieR Online Data Catalog: II/246

Davenport, J. R. A., Kipping, D. M., Sasselov, D., Matthews, J. M., \& Cameron, C. 2016, ApJ, 829, L31

Demory, B.-O., Ségransan, D., Forveille, T., et al. 2009, A\&A, 505, 205

Egan, M. P., Price, S. D., Moshir, M. M., Cohen, M., \& Tedesco, E. 1999, NASA STI/Recon Technical Report N

Egan, M. P., Price, S. D., Kraemer, K. E., et al. 2003, VizieR Online Data Catalog: V/114

Frogel, J. A., Kleinmann, D. E., Kunkel, W., Ney, E. P., \& Strecker, D. W. 1972, PASP, 84, 581

Fröhlich, C. 2012, Surveys in Geophysics, 33, 453

Fuhrmeister, B., Lalitha, S., Poppenhaeger, K., et al. 2011, A\&A, 534, A133

Gautier, III, T. N., Rieke, G. H., Stansberry, J., et al. 2007, ApJ, 667, 527

Gezari, D. Y., Pitts, P. S., \& Schmitz, M. 1999, VizieR Online Data Catalog: II $/ 225$

Gliese, W., \& Jahreiss, H. 2015, VizieR Online Data Catalog: V/35

Güdel, M., Audard, M., Reale, F., Skinner, S. L., \& Linsky, J. L. 2004, A\&A, 416,713

Guinan, E. F., Ribas, I., \& Harper, G. M. 2003, ApJ, 594, 561

Helou, G., \& Walker, D. W. 1988, Infrared astronomical satellite (IRAS) catalogs and atlases, Vol. 7: The small scale structure catalog, 1

Herrero, E., Ribas, I., Jordi, C., et al. 2016, A\&A, 586, A131

Hudson, R. D. 1971, Reviews of Geophysics and Space Physics, 9, 305

Jao, W.-C., Henry, T. J., Subasavage, J. P., et al. 2014, AJ, 147, 21

Jarrett, T. H., Cohen, M., Masci, F., et al. 2011, ApJ, 735, 112

Joint IRAS Science, W. G. 1994, VizieR Online Data Catalog: II/125

Kopp, G., \& Lean, J. L. 2011, Geophys. Res. Lett., 38, L01706

Kopparapu, R. K. 2013, ApJ, 767, L8

Kopparapu, R. K., Ramirez, R. M., SchottelKotte, J., et al. 2014, ApJ, 787, L29

Kopparapu, R. k., Wolf, E. T., Haqq-Misra, J., et al. 2016, ApJ, 819, 84

Lestrade, J.-F., Matthews, B. C., Sibthorpe, B., et al. 2012, A\&A, 548, A86

Lindler, D., \& Heap, S. R. 2008, https://archive.stsci.edu/pub/hlsp/ stisngsl/aaareadme.pdf

Linsky, J. L., Fontenla, J., \& France, K. 2014, ApJ, 780, 61

Lovis, C., Mayor, M., Pepe, F., et al. 2006, Nature, 441, 305
Loyd, R. O. P., \& France, K. 2014, ApJS, 211, 9

Loyd, R. O. P., France, K., Youngblood, A., et al. 2016, ApJ, 824, 102

Luger, R., \& Barnes, R. 2015, Astrobiology, 15, 119

Mamajek, E. E., \& Hillenbrand, L. A. 2008, ApJ, 687, 1264

Mann, A. W., \& von Braun, K. 2015, PASP, 127, 102

Mann, A. W., Feiden, G. A., Gaidos, E., Boyajian, T., \& von Braun, K. 2015, ApJ, 804, 64

Marty, B. 2012, Earth Plan. Sci. Lett., 313, 56

Mould, J. R., \& Hyland, A. R. 1976, ApJ, 208, 399

Mullan, D. J., Mathioudakis, M., Bloomfield, D. S., \& Christian, D. J. 2006, ApJS, 164, 173

Noyes, R. W., Hartmann, L. W., Baliunas, S. L., Duncan, D. K., \& Vaughan, A. H. 1984, ApJ, 279, 763

Passegger, V. M., Wende-von Berg, S., \& Reiners, A. 2016, A\&A, 587, A19

Pecaut, M. J., \& Mamajek, E. E. 2016, MNRAS, 461, 794

Plucinsky, P. P., Beardmore, A. P., Foster, A., et al. 2017, A\&A, 597, A35

Preibisch, T., \& Feigelson, E. D. 2005, ApJS, 160, 390

Press, W. H., Teukolsky, S. A., Vetterling, W. T., \& Flannery, B. P

1992, Numerical recipes in FORTRAN, The art of scientific computing (Cambridge: University Press)

Ranjan, S., \& Sasselov, D. D. 2016, Astrobiology, 16, 68

Redfield, S., Linsky, J. L., Ake, T. B., et al. 2002, ApJ, 581, 626

Reid, N. 1982, MNRAS, 201, 51

Reiners, A., Schüssler, M., \& Passegger, V. M. 2014, ApJ, 794, 144

Ribas, I., Guinan, E. F., Güdel, M., \& Audard, M. 2005, ApJ, 622, 680

Ribas, I., Bolmont, E., Selsis, F., et al. 2016, A\&A, 596, A111

Rieke, G. H., Young, E. T., Engelbracht, C. W., et al. 2004, ApJS, 154, 25

Suárez Mascareño, A., Rebolo, R., \& González Hernández, J. I. 2016, A\&A, 595, A12

Thuillier, G., Floyd, L., Woods, T. N., et al. 2004, Adv. Space Res., 34, 256

Turbet, M., Leconte, J., Selsis, F., et al. 2016, A\&A, 596, A112

Veeder, G. J. 1974, AJ, 79, 1056

Walker, A. R. 1981, MNRAS, 195, 1029

Wargelin, B. J., Saar, S. H., Pojmański, G., Drake, J. J., \& Kashyap, V. L. 2017, MNRAS, 464, 3281

Wood, B. E., Redfield, S., Linsky, J. L., Müller, H.-R., \& Zank, G. P. 2005, ApJS, 159,118

Woodgate, B. E., Kimble, R. A., Bowers, C. W., et al. 1998, PASP, 110, 1183

Wright, N. J., \& Drake, J. J. 2016, Nature, 535, 526

Wright, E. L., Eisenhardt, P. R. M., Mainzer, A. K., et al. 2010, AJ, 140, 1868

Wright, N. J., Drake, J. J., Mamajek, E. E., \& Henry, G. W. 2011, ApJ, 743, 48 